\title{
MHWT-A Modified Haar Wavelet Transformation for Image Fusion
}

\author{
Gurpreet Singh \\ Chandigarh Engineering College \\ Landran, Mohali \\ Punjab, India
}

\author{
Gagandeep Singh \\ Chandigarh Engineering College \\ Landran, Mohal \\ Punjab, India
}

\author{
Gagangeet Singh Aujla \\ Chandigarh Engineering College \\ Landran, Mohali \\ Punjab, India
}

\begin{abstract}
Image Fusion is a process in which combine the relevant or same information from a set of images, into a single image that is more realistic, informative and complete than the previous input images. During the past two decades, many image fusion methods have been proposed and developed. Image Fusion methods are categorized into pixel, feature, and decision levels according to the stage at which image information is integrated. Image fusion algorithms help to achieve benefits like high accuracy and reliability, feature vector with higher dimensionality, faster acquisition of information and cost effective acquisition of information. The proposed technique Modified Haar Wavelet Transform is an enhanced version of Haar Wavelet Transform which can reduce the calculation work and is able to improve the contrast of the image. The main achievement of MHWT is sparse representation and fast transformation. In MHWT at each level, we need to store only half of the original data due to which it becomes more efficient. In this paper we implement Image Fusion MHWT (Modified Haar Wavelet Transformation) and compares its performance with Discrete Wavelet transform (DWT) using performance metrics of standard deviation, entropy and quality index. The modified technique MHWT shows better performance than the earlier methods. A thorough analysis and evaluation of the proposed algorithm is conducted with the help of mathematical formulas
\end{abstract}

\section{Keywords}

Image Fusion, DWT, MHWT, Haar Transform, Wavelet Transform

\section{INTRODUCTION}

In recent times, military and civilian applications, especially in health-care, battlefield surveillance, and traffic control make wide use of imaging sensors. However, the information provided by the different imaging sensors is complementary and redundant [1]. The outline of the scene is provided by visible image, but the actually required special objects, such as concealed guns or people are shown only by infrared image. The medical imaging, microscopic imaging, remote sensing, computer vision [14] and robotics importantly require the successful fusion of images acquired from different modalities or Instruments [7]. Image fusion can be defined as the process by which several images or some of their features of images are combined together to form a single Image of much more importance than the original image. The results of image fusion are intended for presentation to a human observer for easier and enhanced interpretation in areas such as remote sensing and medical imaging[2]. By the process of image fusion the good information from each of the given images is fused together to form a resultant image whose quality is superior to any of the input images. This is achieved by applying some methodology with a sequence of operators on the images that would make the good information in each of the image prominent visible. The resultant image is formed by combining such prominent and magnified information from the input images into a single image [3].

This paper further discusses Image Fusion Algorithms in Section 2 followed by Image Quality metrics in Section 3. The Section 4 shows experimental setup and results with discussion. The Section 5 contains conclusion and future scope.

\section{IMAGE FUSION ALGORITHMS}

The main discussion in this paper aligns along two techniques. The earlier used technique of DWT is compared with the proposed MHWT method. The evolution of the research work into the field of image fusion can be broadly put into the following two stages.

- Wavelet Transformation Method

- Modified Haar wavelet Transformation Method (MHWT)

We implement the proposed modified version of image fusion MHWT (Modified Haar Wavelet Transformation) algorithm and compare it with Wavelet based image fusion algorithm.

\subsection{Wavelet Transformation Method}

Wavelet transform has been greatly used in many areas, such as texture analysis, data compression, feature detection, and image fusion. In this section, we briefly review and analyze the wavelet-based image fusion technique. The DWT can be interpreted as signal decomposition in a set of independent, spatially oriented frequency channels. The signal $\mathrm{S}$ is passed through two complementary filters and emerges as two signals, approximation and Details. This is called decomposition or analysis [4]. The components can be assembled back into the original signal without loss of information. This process is called reconstruction. The mathematical manipulation, which implies analysis and synthesis, is called discrete wavelet transform and inverse discrete wavelet transform. An image can be decomposed into a sequence of different spatial resolution images using DWT. Wavelet based techniques for fusion of 2-D images is described here [6]. In all wavelet based image fusion techniques the wavelet transforms $\mathrm{W}$ of the two registered input images $\mathrm{I}(\mathrm{x}, \mathrm{y})$ and some kind of fusion rule $\varnothing$ as show in below equation.

$$
\mathrm{I}(\mathrm{x}, \mathrm{y})=\mathrm{W}-1\left(\varnothing\left(\mathrm{W}\left(\mathrm{I}_{1}(\mathrm{x}, \mathrm{y})\right), \mathrm{W}\left(\mathrm{I}_{2}(\mathrm{x}, \mathrm{y})\right)\right)\right.
$$

In the case of wavelet transform based fusion method, all respective wavelet coefficients from the input images are combined using the fusion rule $\emptyset$. Since wavelet coefficients having large absolute values contain the information about the salient features of the images such as edges and lines, a good 
fusion rule is to take the maximum of the corresponding wavelet coefficients [8].

\section{Steps of wavelet based image fusion are explained below:}

Step 1: Read the set of multi-focus images i.e. here in our proposed algorithm we have consider two images which are of same size (registered images).

Step 2: Apply wavelet decomposition on both the images with the use of Daubechies filter.

Step 3: Extracts from the wavelet decomposition structure [C, S] the horizontal, vertical, or diagonal detail.

Step 4: Perform average of approximation coefficients of both decomposed images.

Step 5: Compare horizontal, vertical and diagonal coefficient of both the images and apply maximum selection scheme to select the maximum coefficient value by comparing the coefficient of the two images. Perform this for all the pixel values of image i.e. $\mathrm{m} \mathrm{x} \mathrm{n}$.

Step 6: Now apply wavelet decomposition on both the images with the use of Daubechies filter.

Step 7: Display the final fused image.

\subsection{Modified Haar Wavelet Transform}

In Modified fast Haar wavelet transform algorithm after a DWT transform, the image is divided into four corners, upper left corner of the original image, lower left corner of the vertical details, upper right corner of the horizontal details, lower right corner of the component of the original image detail (high frequency) [5].

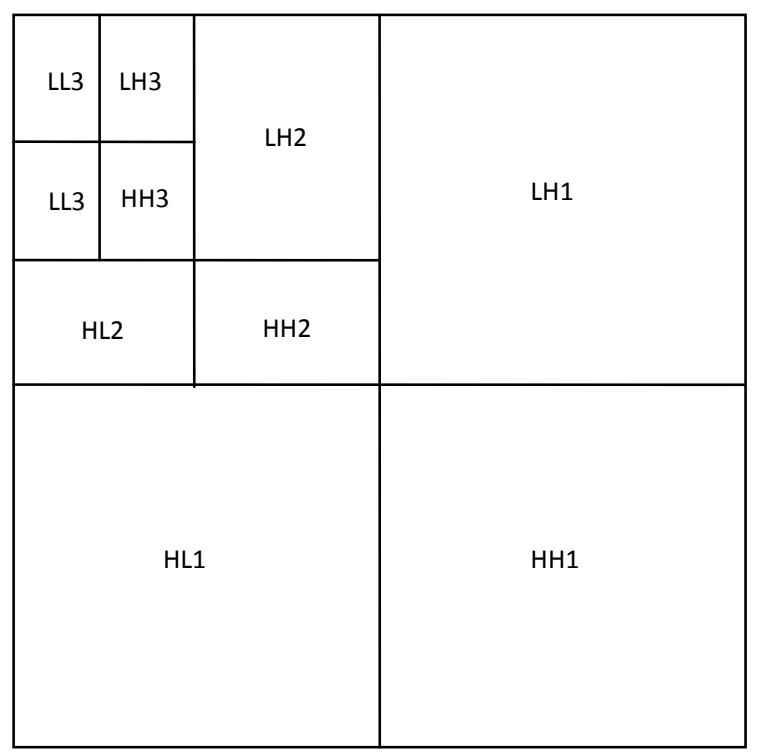

\section{Figure 1: Decomposition of Original Image in Matrix} Form

The LL sub-band (low-low) is in the upper left hand corner and come from low pass filtering in both directions. It is the low resolution residual consisting of low frequency components and will be split at higher level of decomposition. Of the four components, it is the more like the original picture and so called approximation. The remaining three components are called detail components. The upper right corner comes from the high pass filtering in the horizontal direction (low) and low pass filtering in the vertical direction (columns) and so labeled HL. The visible detail in this sub-image, such as, edge, have an overall vertical orientation since there alignment is perpendicular to the direction of the high pass filtering. Consequently they are called vertical details. In MHWT, four nodes are considered at a time instead of two nodes as in DWT. The Figure 1 shows the decomposition of original image in matrix form [9].

In MHWT, four nodes are considered at a time instead of two nodes as in DWT. In MHWT, first average sub signal

signal of length $\mathrm{N}$

$$
a=\left(a_{1}, a_{2}, \ldots . a_{N / 2}\right)
$$

$$
f=\left(f_{1}, f_{2}, f_{3}, f_{4} \ldots \ldots \ldots \ldots \ldots f_{n}\right)
$$

In MHWT, first average subsignal, $\mathrm{a}=(\mathrm{a} 1, \mathrm{a} 2, \ldots, \mathrm{aN} \mathrm{I} / 2)$, at one level for a signal of length $\mathrm{N}$ i.e. is

$$
\mathrm{a}_{\mathrm{m}}=\frac{f_{4 m-3}+f_{4 m-2}+f_{4 m-1}+f_{4 m}}{4}
$$

and first detail sub signal, at the same level is given as

$$
\begin{aligned}
& d_{m}=\left\{\frac{\left(f_{4 m-3}+f_{4 m-2}\right)-\left(f_{4 m-1}+f_{4 m}\right)}{4}, m\right. \\
& =1,2,3, \ldots \ldots, N / 4
\end{aligned}
$$

Here four nodes are considered at a time instead of two nodes as in DWT. The author has considered the values of $N / 2$ detail coefficients zero in each step than to find the N/2 detail coefficients by DWT [13].

\section{MHWT can be done by performing the Following steps}

- Read the image as a matrix.

- Apply MHWT, along row and column wise on entire matrix of the image.

- From (b) we get a transformed image matrix of one level of input image.

- For reconstruction process, DWT is used on the image matrix obtained in step (b).

- Calculate Standard deviation, Entropy and Image Quality index for reconstructed image.

As shown in flow chart in Figure 2 the function developed to perform the image fusion, has four basic blocks:

- Block A: Images size checking. In this Block the size of both the input images are checked. If the sizes of both the images are same then we said both the input images are registered with each other and send them to the next level block B for further processing. If the sizes of both the input images are not same then the algorithm shows the error message and stop processing.

- Block B: Transform to Modified Haar wavelet domain. After registering the both input images with each other we will send these input images to Wavelet domain for further processing.

- Block C: Modified Haar wavelet domain fusion. In this level we decompose the images into some components and applied the Modified haar wavelet function on these components for getting the desired result. 
- $\quad$ Block D: inverse wavelet transforms. After merging all these decompose component with each other we get the final fused Image.

The methodology of MHWT is shown in the below given Figure 2:

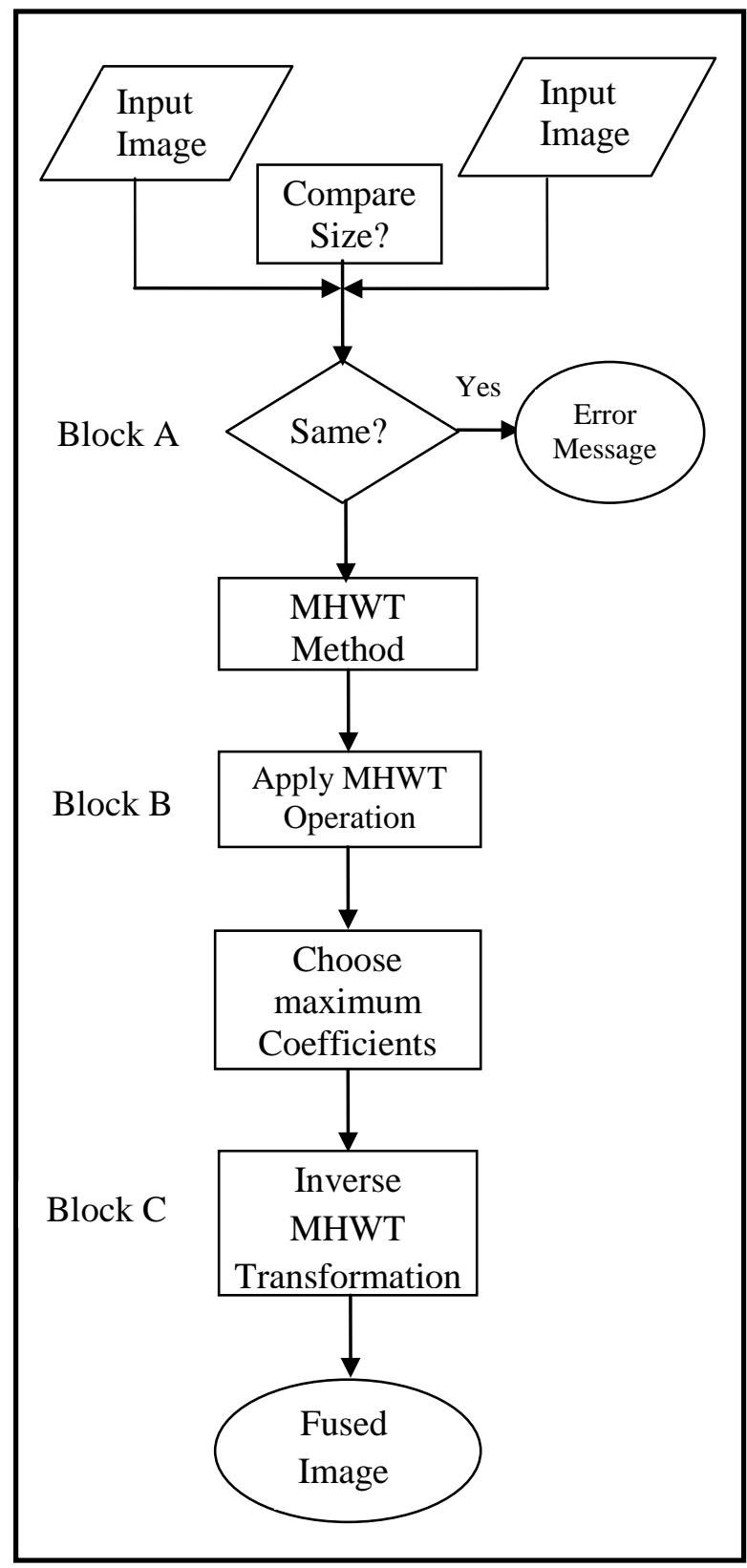

Figure 2: Flow chart of Image Fusion using MHWT

\section{IMAGE QUALITY METRICS}

The Quality metrics are used to measure the performance of the image fusion methods. Image quality is a characteristic of an image that measures the perceived image degradation as compared to an ideal image [12]. The following quality metrics are used in this paper to describe the quality of the image:

Standard deviation (represented by the symbol sigma, $\sigma$ ) shows that how much variation or dispersion exists from the average (mean), or expected value. A low standard deviation indicates that the data points tend to be very close to the mean; high standard deviation indicates that the data points are spread out over a large range of values [10].

Entropy is a statistical measure of randomness that can be used to characterize the texture of the input image [11].

Image Quality index, is easy to calculate and applicable to various image processing applications. This index is designed by modeling any image distortion as a combination of three factors: loss of correlation, luminance distortion, and contrast distortion instead of using traditional error summation methods [12].

\section{EXPERIMENTAL SETUP \& RESULT}

In this section we present the experiments and their results along with their discussions. We fuse the selected sample of input images and the quality of the same was accessed using the quality metrics given in the above section. The test set for this evaluation experiment image randomly selected from the internet. Matlab 7.0 software platform is use to perform the experiment. The PC for experiment is equipped with an Intel P4 2.4GHz Personal laptop and 2GB memory. The scheme is tested using ordinarily image processing. From the simulation of the experiment results, we can draw to the conclusion that this method is robust to many kinds of fused images. The figure 3(a) and 3(b) shows sample images which are to fused.

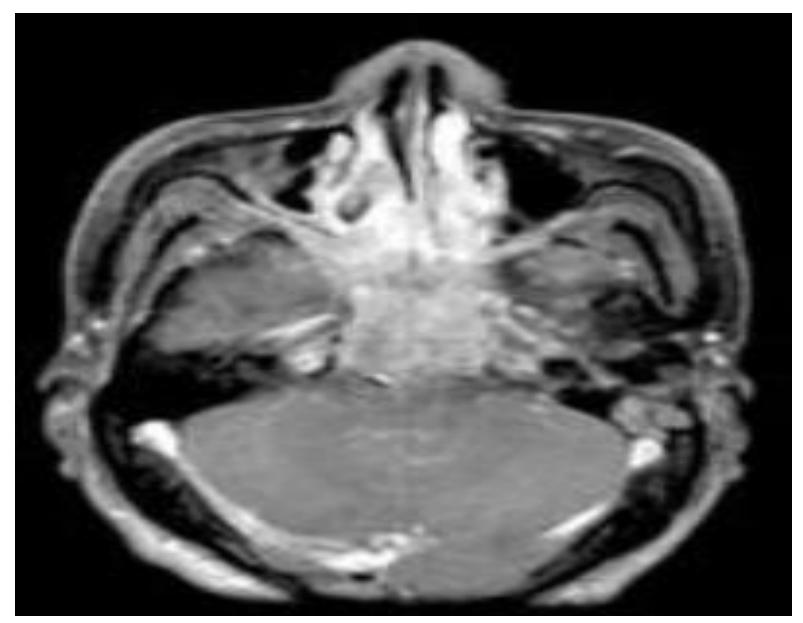

Figure 3(a): Sample Image 1

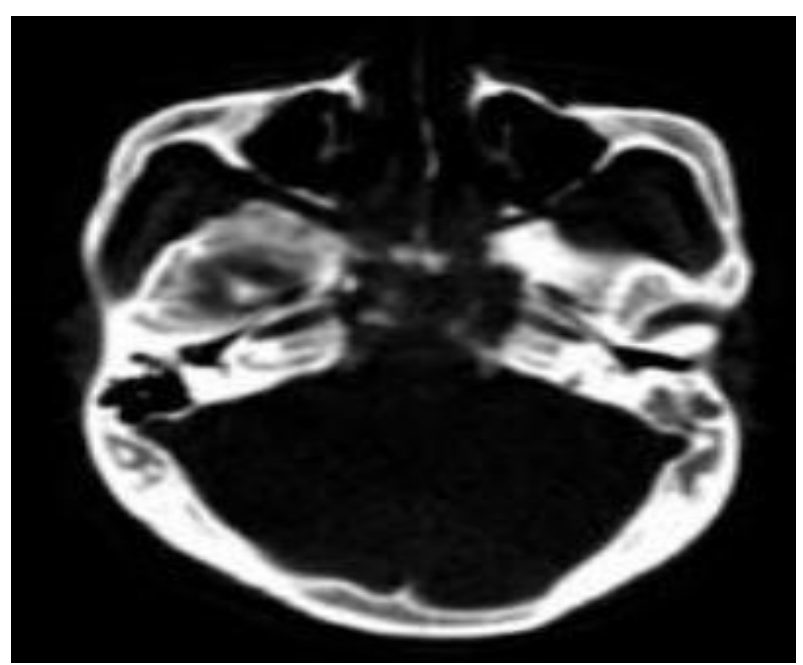

Figure 3 (b): Sample Image 2 
The above two sample images were fused using Matlab 7.0 and the results were used to analyze the quality on the basis of the quality metrics. The resultant images are shown ahead by using both the methods i.e. DWT method and the proposed MHWT method.
First the result of fusion using Discrete Wavelet Transformation fusion method is shown in Figure 4 along with the value of results based on the quality metrics.

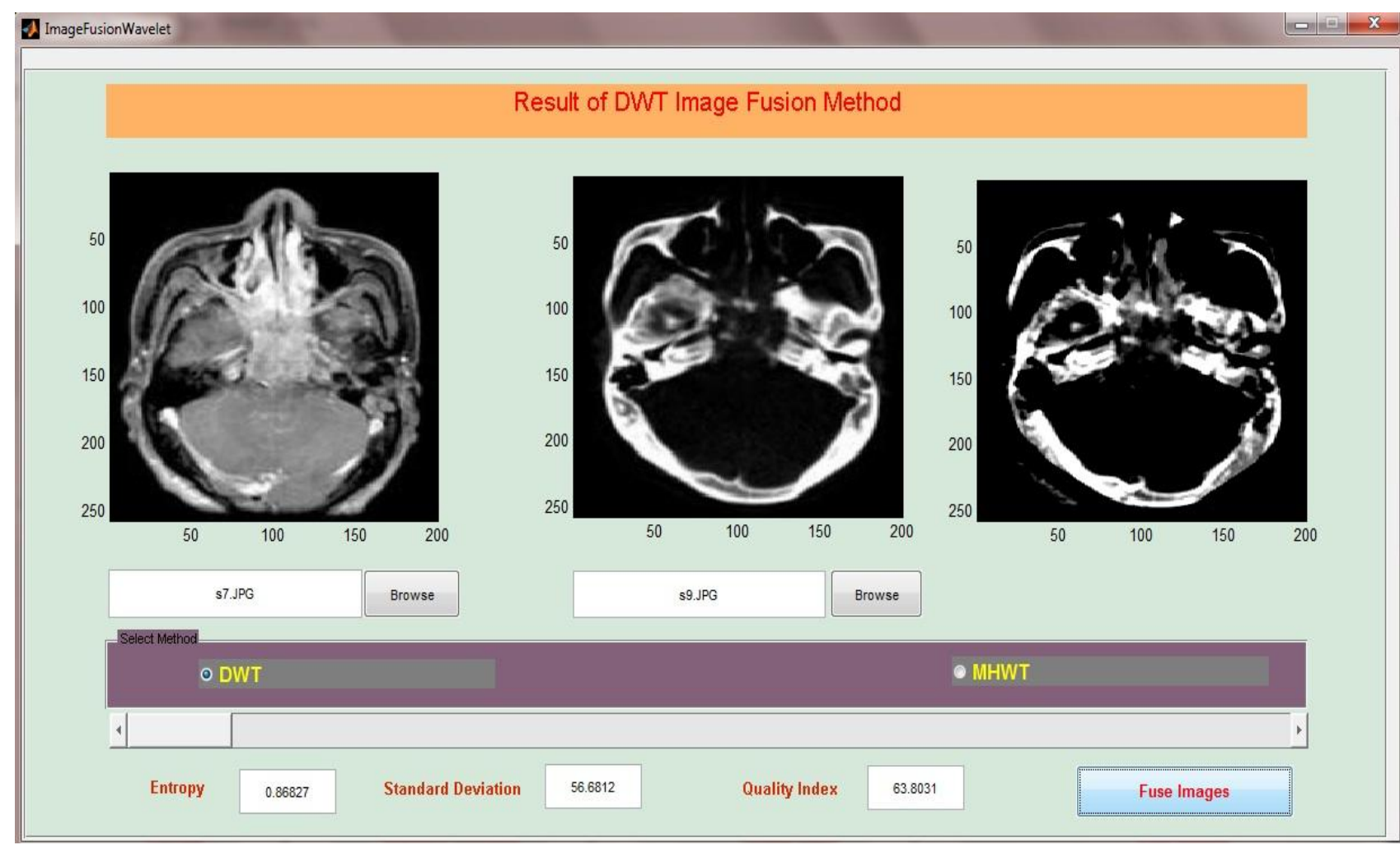

Fig 5: Result of DWT Image Fusion Method

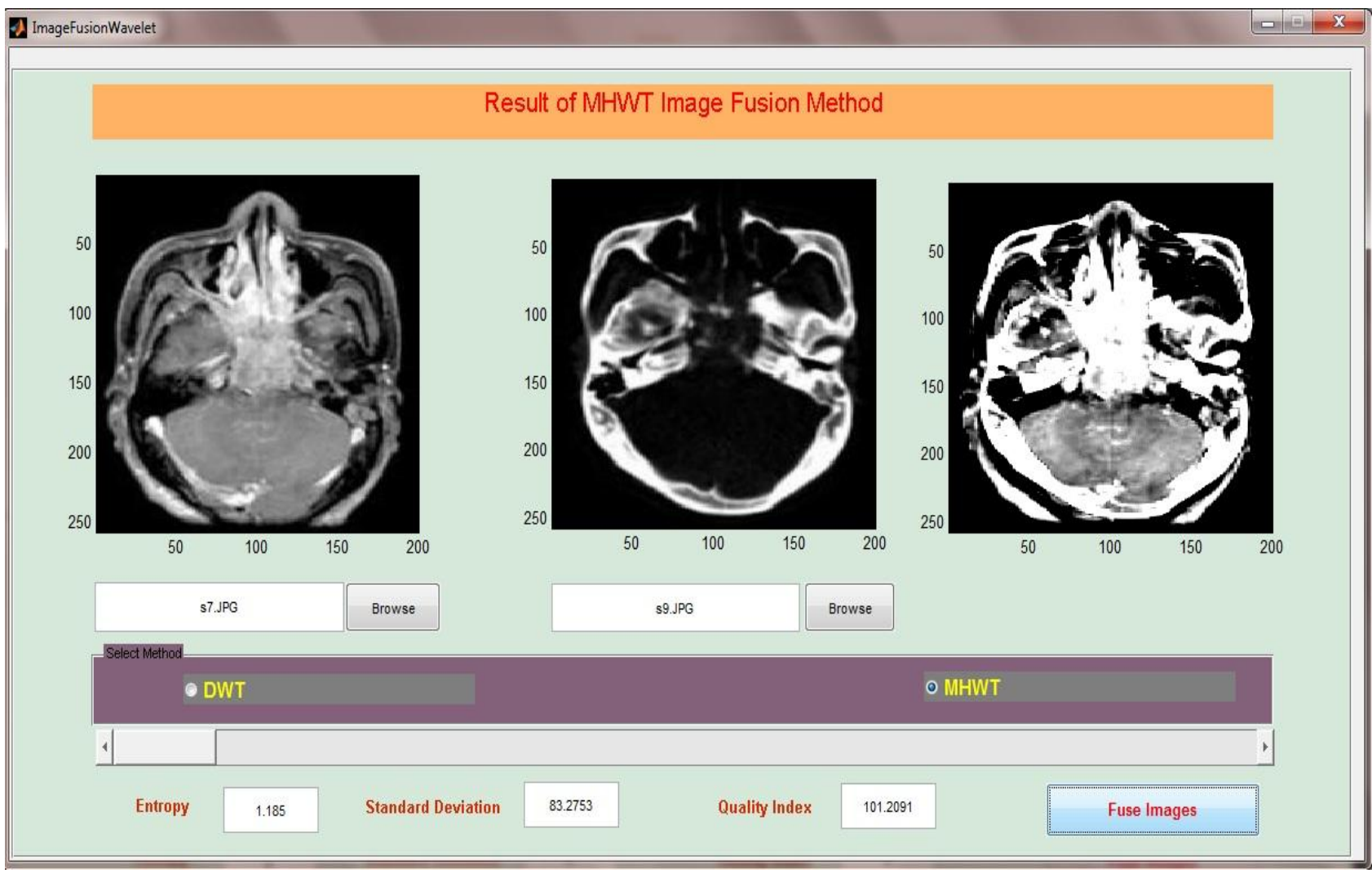

Fig 6: Result of MHWT Image Fusion Method 
The second method used for fusing the two images is MHWT method. The resultant image and the value of quality metrics is shown below in Figure 5.

The value of entropy for Discrete Wavelet Transformation fusion method is 0.86527 and it is less than the value of entropy for MHWT method which is 1.185 as shown in Figure 6 .

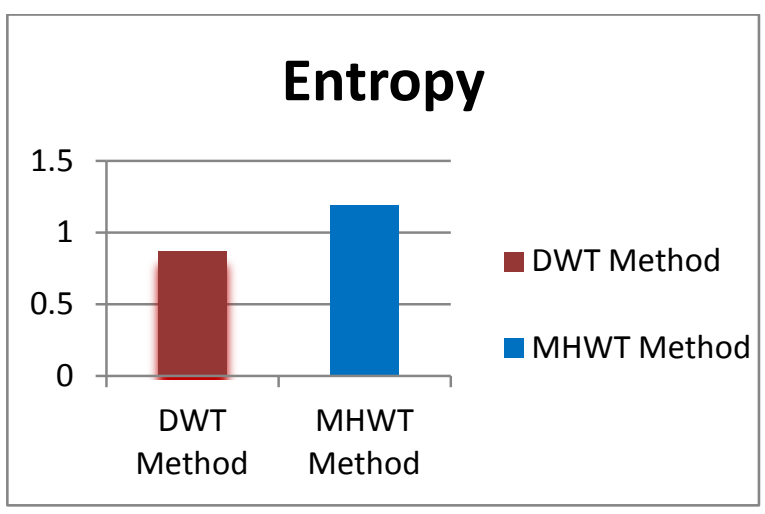

Figure 6: Entropy of DWT and MHWT

The value of standard deviation for Discrete Wavelet Transformation fusion method is 56.6812 and it is less than the value of standard deviation for MHWT method which is 83.2753 as shown in Figure 7.

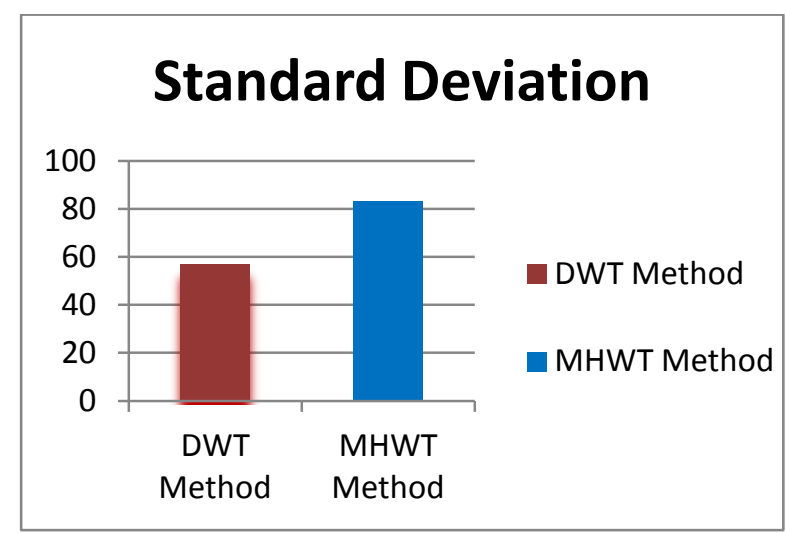

Figure 7: Standard Deviation of DWT and MHWT

The value of Quality Index for Discrete Wavelet Transformation fusion method is 63.8031 and it is less than the value of quality index for MHWT method i.e. 101.2091 as shown in Figure 8.

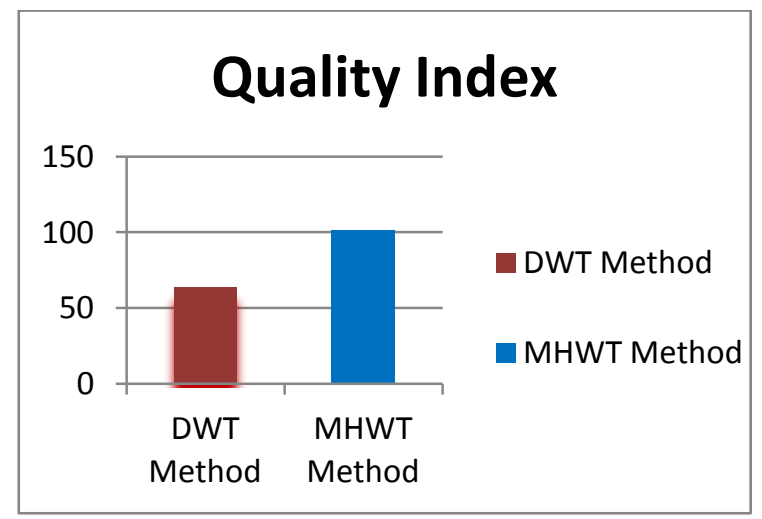

Figure 8: Quality Index of DWT and MHWT
The below given Table 1 shows the comparison of Discrete Wavelet Transform method and the proposed MHWT method. The below given table enable us to analyses the image fusion results with better perception.

Table 1: Result analysis of DWT and MHWT method

\begin{tabular}{|c|c|c|}
\hline $\begin{array}{c}\text { Performance } \\
\text { Metrics }\end{array}$ & DWT Method & MHWT Method \\
\hline Entropy & 0.86527 & 1.185 \\
\hline Standard deviation & 56.6812 & 83.2753 \\
\hline Quality Index & 63.8031 & 101.2091 \\
\hline
\end{tabular}

\section{CONCLUSION \& FUTURE SCOPE}

In this paper we have experimentally analyzed two image fusion techniques namely Discrete Wavelet Transform method and MHWT (Modified Haar Wavelet Transformation) method using Entropy, Standard Deviation and Quality Index Image Metrics. These experiments were conducted using Matlab 7.0. Depending upon the purpose of a given application, (1) some users may desire a fusion result that shows more detail in color, for better image interpretation or mapping; (2) some may desire a fusion result that improves the accuracy of digital classification; and (3) some others may desire a visually beautiful fused color image, solely for visualization purposes. Therefore, distinct techniques for mapping oriented fusion, classification-oriented fusion, and visualization-oriented image are in demand.

Following conclusions have been drawn from experimental results:

- MHWT (Modified Haar Wavelet Transformation) have better Image quality Index as compare the DWT Image Fusion Method

- MHWT (Modified Haar Wavelet Transformation) have better Standard deviation as compared the DWT Image Fusion Method

- MHWT (Modified Haar Wavelet Transformation) have better Entropy as compared the DWT Image Fusion Method

The overall evaluation shows that MHWT method is far better than DWT image fusion method.

In future study we can combine Hybrid technique (MHWT and Pixel by Pixel) to achieve better image fusion result than the previous methods that we have discussed.

\section{REFERENCES}

[1] Krishnamoorthy, S. and Soman, K. P. (2010), "Implementation and Comparative Study of Image Fusion Algorithms", International Journal of Computer Science \& Communication, Volume 9- No.2, November 2010.

[2] Praveena, S. M. and Vennila, I. L. A. (2009) "Image Fusion By Global Energy Merging", International Journal of Recent Trends in Engineering, Vol 2, No. 7 , November 2009.

[3] Naidu, V.P.S. and Raol, J.R. (2008) "Pixel-level Image Fusion using Wavelets and Principal Component Analysis", Defence Science Journal, Vol. 58, No. 3, May 2008, pp. 338-352 Ó 2008, DESIDOC. 
[4] Solanki, C. K. and Patel, N. M. "Pixel based and Wavelet based Image fusion Methods with their Comparative Study", National Conference on Recent Trends in Engineering \& Technology.

[5] Rao, M.J.M. and Reddy, K.V.V.S. (2011) "Image Fusion Algorithm for Impulse Noise Reduction in Digital Images", Global Journal of Computer Science and Technology, Volume 11 Issue 12 Version 1.0 July 2011.

[6] Prakash, N. K. (2011) "Image Fusion Algorithm based on Bioorthogonal Wavelet", International Journal of Enterprise Computing and Business Systems, Vol. 1 Issue 2 July 2011

[7] Pohl, C. and Genderen, J. 1. V. (1998) "Multisensor image fusion in remote sensing: concepts, methods and applications", International Journal of Remote Sensing, 1998, vol. 19, no. 5, 823-854.

[8] Asmare1, M. H., Asirvadam, V. S., Iznita, L. and Hani, A. F. M.(2010) "Image Enhancement by Fusion in Contourlet Transform", International Journal on Electrical Engineering and Informatics, Volume 2, Number 1, 2010

[9] Malviya, A, Bhirud, S. G.(2009) "Image Fusion of Digital Images", International Journal of Recent Trends in Engineering, Vol 2, No. 3, November 2009.
[10] Pati, U. C., Dutta, P. K. and Barua, A.(2010) "Feature Detection of an Object by Image Fusion", International Journal of Computer Applications, Volume 1 - No. 1.

[11] Nunez, J, Otazu, X, Fors, O, Prades, A, Pala, V and Arbiol, R. (1999) "Multiresolution-Based Image Fusion with Additive Wavelet Decomposition," IEEE Transactions On Geoscience and Remote Sensing, vol. 37, no. 3, May 1999

[12] Chipman, L.J., Orr, T.M, and Lewis, L.N. et al., (1995). "Wavelets and image fusion". Proceedings of IEEE International Conference on Image Processing, Volume 3 , pages $248-251$

[13] Li, H, Manjunath, BS and Mitra, S. (1994) "Multi-Sensor Image Fusion Using Wavelet Transform". Proceedings of the IEEE International Conference on Image Processing, vol. 1, pp. 51-55.

[14] Burt, P.J. and Kolczynski, R.J. 1993 Burt, P.J. and Kolczynski, R.J. (1993). "Enhanced image capture through fusion". Proceedings of the $4^{\text {th }}$ International Conference on Computer Vision, Pages 173-182. 